\title{
Wer spricht in einer Literaturausstellung?
}

\author{
Überlegungen zum dialogischen Möglichkeitsraum \\ einer Gattung, angestoßen von Helmut Neundlinger
}

„Wer spricht hier eigentlich?“ Was mich an dieser Frage umtreibt, die Helmut Neundlinger $^{1}$ mir als Kuratorin der Ausstellung im Schiller-Nationalmuseum unerwartet direkt gestellt hat, das ist nicht: Wie wird ein ,Ich' und gar mein ,Ich“ sichtbar - das lässt sich leicht durch eine Personalisierung der Texte leisten -, sondern: Was ergibt sich an Möglichkeiten, wenn man offensiv mit dieser Grundvoraussetzung von Ausstellungen umgeht: Hier sprechen Einzelne mit den Besuchern über Gegenstände? Was ergibt sich an Möglichkeiten, wenn man diese Grundvoraussetzung an das Ziel einer Ausstellung zurückbindet: Diese Einzelnen tun das, weil sie jedem einzelnen Besucher an ihren Gegenständen etwas zeigen und ihn dazu anstiften möchten, selbst und freiwillig mit diesen Gegenständen in einen Dialog zu treten. Die kuratorische Stimme ist nicht selbstbezüglich und singulär, sondern an andere gerichtet, in all ihrer Eigenwilligkeit.

Die Frage nach dem, was eine Literaturausstellung kann, ist umso virulenter, weil allmählich zwei andere Vorstellungen, die traditionellerweise zum Erwartungshorizont einer Literaturausstellung gehören, durch das Internet und für alle zugängliche Online-Kataloge und -Lexika obsolet werden: die Vermittlung eines objektiven Wissens und das Zeigen von Originalen als Dokumente dieses Wissens. Warum daher nicht die bestehenden Vorstellungen der Besucher unterlaufen und das stärken, was die Stärken der Gattung ,Ausstellung' sind - das Erfahren von eigenen, gedanklichen und emotionalen, aber auch körperlichen Singularitäten, von Abständen und Schwellen in einem öffentlichen, ästhetisch definierten Raum?

Brisant wird diese Frage in unserer gegenwärtigen politischen Situation, in der Menschen verallgemeinert und mit Kategorien wie ,Gruppe“, ,Mehrheit‘, ,Minderheit‘, ,Leitkultur‘, ,Andere‘, ,Fremde‘ oder den vermeintlichen Gegenbegriffen ,Bevölkerung‘, ,Integration', ,Inklusion' und ,Beteiligung“ operiert wird, wie sie auch im Kulturmanagementbereich sehr gern verwendet werden. Was geschieht, wenn wir in Literaturausstellungen diese Kategorien schlicht auflösen und nur eines setzen: Es geht in ihnen wie in der Literatur um lauter Einzelne. „Wer sich mit dem Erzählen von Geschichten beschäftigt“, sagte Christoph Ransmayr 2014,

1 Siehe dazu den Beitrag von Helmut Neundlinger in diesem Band.

Ә Open Access. (c) 2019 Heike Gfrereis, publiziert von De Gruyter. (c) BY-NC-ND Dieses Werk ist lizenziert unter der Creative Commons Attribution-NonCommercial-NoDerivatives 4.0 Lizenz. 
als er den Theodor-Fontane-Preis der Stadt Neuruppin erhielt, „der hat es immer nur mit dem Einzelnen zu tun“:

nie mit ganzen Stämmen, Völkern oder gar der jüngsten und dümmsten Perversion der Horde, der Nation ..., wer sich also mit dem Einzelnen, mit Namen und Anschrift beschäftigt, seinem Glück, Wahn oder Leiden, der wird im Leben der anderen, selbst im Schicksal seiner erfundenen Figuren immer auch etwas von seinem eigenen Leben zur Sprache und zum Schwingen bringen und er wird, in dem er den Spuren nachzugehen versucht, die unseresgleichen hinterlassen hat und hinterläßt, immer auch dem nachgehen, was ihn selbst und uns bewegt oder zumindest in Bewegung versetzen könnte. (Ransmayr 2018, 78)

Was passiert, wenn wir erkennen, dass die Realität (nicht nur einer Ausstellung) vor allem durch unsere gefühlte Wahrheit bestimmt wird und damit immer auch unser eigenes Konstrukt ist? „Wie alle Bücher ist auch das vorliegende Buch von dem Begehren angetrieben, etwas überleben zu lassen, Vergangenes zu vergegenwärtigen, Vergessenes zu beschwören, Verstummtes zu Wort kommen $\mathrm{zu}$ lassen und Versäumtes zu betrauern“, schreibt Judith Schalanksy in ihrem Verzeichnis einiger Verluste:

Nichts kann im Schreiben zurückgeholt, aber alles erfahrbar werden. So handelt dieser Band gleichermaßen vom Suchen wie vom Finden, vom Verlieren wie vom Gewinnen und lässt erahnen, dass der Unterschied zwischen An- und Abwesenheit womöglich marginal ist, solange es die Erinnerung gibt. Und für wenige kostbare Momente erschien mir während der langjährigen Arbeit an diesem Buch die Vorstellung, dass das Vergehen unvermeidlich ist, genauso tröstlich wie das Bild seiner in Regalen verstaubenden Exemplare. (Schalansky 2018, 26)

Meine Antworten möchte ich in zehn Thesen zuspitzen, die wiederum in einige Fragen münden, die vielleicht zu einer neuen Art führen könnten, Literaturausstellungen zu denken und zu realisieren.

\section{1}

Die Vorstellung, Literaturausstellungen wären Schaufenster eines Archivs, ist mit der digitalen Zugänglichkeit von Beständen ebenso überholt wie die Vorstellung, Literaturausstellungen vermittelten Wissenszusammenhänge (wie z. B. eine Einführung in Leben und Werk, die für alle frei zugänglich im Internet lesbar sind). Es gibt nichts, was sozusagen jenseits der Archiv-, der Wissens- oder auch der Literatur-Schnittstelle ,Ausstellung schon selig in sich selbst scheint und durch die Ausstellung nur zum Vorschein gebracht wird. In Wahrheit ist es tendenziell andersherum: Die Sachen des Archivs bringen wie literarische Texte in uns etwas 
zum Vorschein. Wir als Einzelne beschäftigen uns mit ihnen als Einzelne. Indem die Wissensvermittlung durch andere Medien abgenommen wird, können Ausstellungen Orte des offenen Denkens und der besonderen (Selbst-)Erfahrungen werden.

\section{2}

Georges Bataille bestimmte 1929 die „Räume und Kunstgegenstände in einem Museum“ als „Gefäß“: „Den Inhalt bilden die Besucher“ (Bataille 2005, 64). Kuratoren sind dann nichts anderes als erste Besucher; sie leiten an zum Hin- und Herdenken von und mit Gegenständen im Raum. Wobei alles ein Gegenstand sein kann: eine Archivalie ebenso wie ein gefakter Alltagsgegenstand, ein inszenierter Text, ein Raum, eine Idee, ein Ton, eine Lichterscheinung an der Wand ...

\section{3}

Wenn Kuratoren erste Besucher sind, dann dürfen und müssen sie ihre eigene Stimme haben. Sie müssen den Mut haben zu autorisieren, zu provozieren, zu reflektieren, aber auch zur Verantwortung gezogen zu werden und $\mathrm{zu}$ diskutieren, sich zu öffnen, weiter zu gehen, auch noch einmal anders zu denken. Alle wissen: Es ist nur die Stimme eines Einzelnen oder mehrerer Einzelner im Dialog, die just in diesem Augenblick der Ausstellung ihren Ton, ihre Linie, ihre Haltung und Rolle gefunden hat. Viele andere Stimmen sind denkbar und möglich. Kuratieren ist so immer auch eine spezielle Form der Kritik an der Ausstellung, die mit der Eröffnung nicht fertig ist, sondern erst ihr Eigenleben beginnt, und die schon vor der Eröffnung ihr Begleitprogramm in den Prozess der Ausstellungsentstehung verlegt.

\section{4}

Ausstellungen, die einen Autor haben, sind von vornherein klar gesetzt, sie sind relativ, nicht absolut, was heißt: Sie müssen diese Relativität nicht durch einen absoluten Gestaltungseffekt überstrahlen und hinter ihm verstecken. Ausstellungsgestaltung darf relativ sein, ironisch, minimal ... Sie darf Leerräume lassen. Sonst geht eine unmittelbare, für die Gattung ,Ausstellung، wichtige Erfahrung verloren. Eine Ausstellung ist eine individuelle Setzung auf Zeit, ein Umgehen mit einem bestimmten Raumformat, dessen Form und Struktur dann am deut- 
lichsten sind, wenn es leer ist. Ob es mehr Bühne ist oder mehr Luftraum oder mehr Raummaß oder Spielwiese, hängt vom Einzelfall ab. Dieses Raumformat ist an kein Museum gebunden, an keinen festen Ort. Es kann an allen Orten definiert werden. Mit dem einfachen Mittel, das die Straßenkünstler dafür haben: mit Kreide einen Kreis ziehen. Pop-Up-Ausstellungen kommen überall hin. ${ }^{2}$

\section{5}

Da Ausstellungen durch Abstände im Raum definiert werden, lassen sie unterschiedliche Stimmen zu: Meinungen können gegeneinanderstehen. Sie brauchen nicht zwingend den Kompromiss und sie dürfen sich entwickeln, dürfen freier und auch ein wenig verrückter werden. Aber sie können den Kompromiss auch thematisieren, als eine Ausstellung, die Probleme kollektiven Ausstellens sichtbar macht und damit auch die Probleme demokratischer Prozesse entdeckt, aufzeigt, benennt und reflektiert. Ausstellungen sind kein abgeschlossenes Kunstwerk. Und sie sind nicht die sinnliche Inszenierung eines Masternarrativs, das in einfacher und verkürzter Form in die Pressemitteilung übersetzt werden kann und in der Flut der unterschiedlichen Dinge, Wahrheiten und Welten beruhigt: Es gibt sie noch, die einfachen Wahrheiten ... ${ }^{3}$ Ausstellungen brauchen eigene und besondere, individuelle Sprachen. Ausstellungen dürfen dem, der sie macht, Spaß machen. Es geht um das Einzelne, persönlich Richtige, die Erfahrung von etwas, was man noch nicht kennt, und nicht um das richtige Allgemeine und das

2 Zum Begriff „Pop Up Museum“, hinter dem mehr steckt als eine Instagram-Aktion vgl. Grant (2018).

3 Und so zu den immer selben Redewendungen führt, die Christian Demand am Beispiel von Arsprototo, dem Magazin der Kulturstiftung der Länder, beschreibt: „Der beflissen kennerschaftliche Duktus der Textbegleitung, die sich gern in Manufactum-Manier durch onkelhaften Renommierton (,erlesen', ,veritabel', ,unikal', ,gebührend', ,delikat', ,beredt') aufspreizt [...]. Worüber auch immer die Zeitschrift berichtet, man kann darauf wetten, es ist ,einzigartig' und / oder ,legendär‘: Einzigartig sind die Sammlungen, von denen die Rede ist, einzigartig ihre Qualität, einzigartig ihre Wirkung, einzigartig schließlich auch der Glücksfall, der die Erwerbung oder auch Instandsetzung möglich machte. Legendär sind Ausstellungen, Galeristen, Sammler, Kunsthistoriker. Exponate wiederum sind wenigstens ,hochkarätig‘ (,Fürstenkultur vom Feinsten'). Werke ,strahlen' selbstverständlich, und sind, sofern sie nicht ohnehin als ,Meisterwerk', ,Hauptwerk', ,Schlüsselwerk‘, ,Rarissimum‘, ,Bravourstück‘, ,Prachtstück‘, ,Schmuckstück', ,Glanzstück', ,wahrhafter Schatz' und damit als ,erlesen' gelten können, doch praktisch immer ,herausragende Zeugnisse‘, ,außergewöhnlich‘, ,zentral', ,bedeutend‘, ,grandios‘, ,kostbar‘ oder zumindest ,wichtig', und spiegeln deshalb ,auf unnachahmliche Weise‘ den Geist ihrer Zeit wider“ (Demand 2018, 18-20). 
Erfüllen einer Form. Wer eine Ausstellung kuratiert, der muss nicht durch eine benotete Prüfung, so wenig wie die Besucher nachher geprüft und benotet werden. Reizvoller wären am Ende diese Fragen: Was habt Ihr nicht verstanden? Was könnt Ihr nicht in Sprache übersetzen? Wo seid Ihr ganz anderer Meinung? Oder: Was blieb Euch in Erinnerung? Was werdet Ihr nicht vergessen? In welcher Stimmung seid ihr?

\section{6}

Eine Ausstellung zu denken und zu gestalten und zu vermitteln, heißt dann nicht: Inhalte $\mathrm{zu}$ formatieren und Brüche $\mathrm{zu}$ glätten, sondern sie profiliert in den Raum zu stellen und Besucher mit ihr zu konfrontieren. Von der ersten Idee bis zur letzten Führung ist Ausstellungenmachen für alle Beteiligten ein Dialog, ein Weiterentwickeln und Ändern, keine Übersetzung oder Umsetzung von Vorgaben. Um offen sein zu können, braucht es Planungs- und Finanzierungssicherheit. Gestaltung ist daher keine späte Zutat, sie schafft für dieses dialogische Vorgehen die Grundlagen. Von jedem Einzelnen fordert der Dialog, dass er sich einbringt, mit dem, was er besonders kann, und nicht mit dem, was alle können sollen - mit dem also, was sich durch die Sache, das gemeinsame inhaltliche Anliegen aller Beteiligten sowie ein Verständnis für die Bedingungen und Eigenheiten der Gattung ,Ausstellung' motivieren lässt und nicht mit der Struktur einer Institution. Im idealen Fall werden dabei dann auch die Widerstände offen verhandelbar, auf die Ausstellungen bei ihrer Realisierung stoßen, die von der Form ihres Ablaufs und vom Einhalten von Formalia aus gedacht werden (die aber im öffentlichen Bewusstsein wie in der Literaturausstellungskritik keine Rolle spielen, obwohl sie für die Gattung zentral sind, wie Geld z. B., Urheberrechtsfragen oder auch immer wieder neu gesetzte und verschärfte konservatorische Auflagen, die inzwischen die Erscheinung und Wirkung jeder Literaturausstellung mit Originalen maßgeblich prägen - wenn die Mittel dafür vorhanden sind: Geld, Verdunklungs- und Klimatisierungsmöglichkeiten, und es ein Autor wie Günter Grass, der sich für seine Bilder und Skulpturen Tageslicht wünschte, nicht explizit anders verfügt hat).

\section{7}

Wenn der Inhalt einer Ausstellung die Besucher sind, dann sind diese immer auch deren Gegenstand und Teil. Was man ihnen deutlich sagen sollte und in was man sie aktiv einbeziehen kann, indem die Ausstellungen responsiver werden, 
auf Besucher reagieren und ihnen etwas über sich erzählen, weil sie z. B. deren Reaktionen provozieren und sich durch sie verändern oder diese dann auch messen, dokumentieren, erfragen und evaluieren.

\section{8}

Pluralität ist heute längst nicht mehr nur eine ästhetische Qualität (,je ne sais quoi'), sondern ein politischer, immer wieder aufs Neue zu erobernder Wert. Was bedeutet: Pluralität schmälert nicht das Diktat der Moderne, das mit der Singularität untrennbar verbunden ist - die Originalität des Einzelnen. Im Gegenteil: Pluralität lässt Originalität erst zu.

\section{9}

Literaturausstellungen sind unter diesen Umständen soziale Räume und Modelle für Denk- wie Aushandlungsprozesse in einer geschützten Zone. Sie sind keine Gattung mehr, die durch Kanon- und Gegenkanonkriterien orientierte Sammlungen, statische Narrative, feste Raumbilder und illustrative Übersetzungen geprägt ist, sondern der Schauplatz, an dem auf ästhetische Weise über Fantasien und Gefühle, Begriffe und Werte debattiert wird.

\section{0}

Das Grundprinzip des Ausstellens, d. i. das Prinzip der nicht-natürlichen Kombination von Gegenständen im Raum, für die bei allen Ähnlichkeiten Unterschiede konstitutiv sind, ist ein soziales Modell für ein gleichberechtigtes und dialogisches Nebeneinander von unterschiedlichen Perspektiven, ein soziales Modell für das gewaltfreie Umgehen mit Symmetrien und Asymmetrien: „Ein Dialog ohne semiotische Differenz ist sinnlos, wenn die Differenz jedoch ausschließlich und absolut wird, so ist kein Dialog mehr möglich. Asymmetrie setzt ein gewisses Maß an Invarianz voraus“ (Lotman 2010, 191). Eine Ausstellung ist der ideale Proberaum für eine Erfahrung, die mit der Literatur extrem verbunden ist: sich auf etwas einzulassen, was man nicht selber ist und was erst in dem Maß entsteht, in dem man sich darauf einlässt. Wobei die Vorzeichen immer klar sind, weil das Gegenüber dabei aus Schrift und Papier besteht: Man gibt sich selbst nicht auf, wenn man sich auf etwas anderes einlässt. Die reale Differenz bleibt trotz der ästhetischen Resonanz bestehen: 


\begin{abstract}
Eine pluralistische Diskussionskultur entsteht aus der blossen Vielzahl an Einstellungen nämlich erst dann, wenn diese einen gemeinsamen Resonanzraum haben, wenn sie sich nicht bloss voneinander abheben, sondern auch offen miteinander auseinandersetzen. Mit anderen Worten: wenn Meinungen irritierbar bleiben. Einen Mangel an divergierenden Standpunkten jedenfalls dürften derzeit die wenigsten konstatieren. Viel eher schon eine Vulgarisierung des Protests, eine tiefe Krise des Zuhörens und die aktive Selbstabschottung gegen jede bessere Einsicht. Wo aber der Einspruch gegen die abweichende Meinung lediglich die eigene verabsolutieren will, geschieht das Gegenteil von Kritik. Entgegen einem landläufigen Verständnis von Kritik als blossem (Negativ-)Urteil versteht sich Kritik in langer philosophischer Tradition als die Tätigkeit, durch die sich ein begründetes Urteil überhaupt erst bildet. Kritik ist also keine aggressive Ansage, sondern ein Prozess. Sie ist zunächst eine Praxis des Differenzierens und versagt sich das Voreilige ebenso wie das Endgültige - denn jedes ihrer Ergebnisse kann selbst wieder Gegenstand von Kritik werden. [...] Die Beschäftigung mit Literatur ist eine Vorzeigeschule im Umgang mit Mehrdeutigkeit und Kontingenz. Wenn ein Kernproblem unserer gegenwärtigen Diskussionskultur die ideologische ,Vereindeutigung der Welt‘ ist [...], dann stellen die Ambivalenzerfahrung und das Rollenspiel der Literatur dazu ein Gegenmodell bereit. Was sich also für die Debattenkultur von der Literatur lernen liesse, ist nicht eben wenig: Anti-Dogmatismus zum Beispiel und Komplexitätstoleranz. Fantasie und Möglichkeitsdenken statt Alternativlosigkeit und Engstirnigkeit. (Graf 2018)
\end{abstract}

Daraus ergeben sich andere Fragen. Zum Beispiel: Wie kann Kritik Teil einer Ausstellung werden, treibendes Element eines Prozesses und eben nicht nur ein einmaliges Urteil über sie fällen? Was kann möglicherweise tatsächlich nur im Kunstraum einer Ausstellung gesehen und erfahren, gedacht, erforscht und gesagt werden, mit allen offenen Widersprüchen, Polyphonien und Ambivalenzen? Wie wirken ästhetische, literarisch-künstlerische oder philosophisch-theoretische Texte im öffentlich-ästhetischen Raum auf uns? Wie erweitern sich die Grenzen eines traditionellen, an die Schrift und das Buch gebundenen Textbegriffs, wenn man die Medien wechselt und statt von der Textualität und Materialität von ästhetisch-poetischen Erfahrungen und den realen, persönlichen, politischen und sozialen Wirkungen von Literatur ausgeht? Was geschieht, wenn Ausstellungen mit schnellen, offenen, auf kleine, erreichbare Ziele hin angelegten, hackathonähnlichen Erarbeitungsweisen von Anfang an als bewegliche Werkstatträume konzipiert und entworfen werden? Was, wenn sie von künstlerischforschenden Bespielungen wie empirisch-psychologischen Besucherforschungen begleitet und ihrerseits stimuliert werden? Wie halten Besucher Polyphonien und Widersprüche aus, die ihnen ja durchaus selbst Zeit und Aufmerksamkeit und Einsatz abverlangen? Lassen sich Erfahrungen von Negativität (wie z. B. Verneinungen, Enttäuschungen, Unverständlichkeit, Trauer und Schrecken) in einer Ausstellung als Teil einer ästhetischen Erfahrung positiv empfinden und kann 
dieser positive Umgang mit Negativität z. B. durch empirische Einzelfall-Untersuchungen ${ }^{4}$ und Gespräche verstärkt und reflektiert werden?

Vorerst sind das Fragen. Gedankenspiele. Und sie sollten es in einer Hinsicht bleiben, selbst dann, wenn in Zukunft das eine oder andere Wirklichkeit wird, weil es mir das Beste an unserem Gegenstand, an unserer Sache - der Literatur im Raum, die dort anders als zu Hause auch mit dem Körper und in der Bewegung erfahren wird, nicht nur im Lesepakt, der zwischen Hand und Kopf besteht $-\mathrm{zu}$ sein scheint: Gedankenspiele, Sprachspiele eröffnen reale Möglichkeitsräume, in denen jede und jeder sprechen darf und es ein paar nur vormachen, wie man sprechen könnte, um den falschen Respekt vor der Kunst zu nehmen. Kunst ist nie Systemerfüllung, sondern immer auch Systemüberschreitung. Sie ist unter Bedingungen der Demokratie eine Möglichkeit, die eigene Wahrheit zu sagen oder zu zeigen und ebenso: frei, ohne Lüge und Peinlichkeit zu phantasieren. Agitation ist Kunst zwangsläufig nur unter anderen, nicht mehr demokratischen Bedingungen, die Bertolt Brecht 1938 im Pariser Exil benannt hat:

Wer heute die Lüge und Unwissenheit bekämpfen und die Wahrheit schreiben will, hat zumindest fünf Schwierigkeiten zu überwinden. Er muss den Mut haben, die Wahrheit zu schreiben, obwohl sie allenthalben unterdrückt wird; die Klugheit, sie zu erkennen, obwohl sie allenthalben verhüllt wird; die Kunst, sie handhabbar zu machen als eine Waffe; das Urteil, jene auszuwählen, in deren Händen sie wirksam wird; die List, sie unter diesen zu verbreiten. (Brecht 1990, 222)

Ausstellungen sind eine Gattung, in der sich etwas zeigt. Etwas, was nicht sichtbar war oder sonst nicht sichtbar wäre, wird enthüllt und noch mehr: Es wird so gezeigt, dass sich daran eine Vielzahl von Blickwinkeln eröffnen. Ausstellungen sind nicht, mit was sie aber oft verwechselt werden, ein Ort der Repräsentation, an dem sich in den Gegenständen etwas Drittes zeigt. Die Ausstellungssprache rührt auf eine Weise an die Gegenstände, dass sie sich zeigen, und nicht nur etwas repräsentieren. Ihre Grenzen zur Poesie sind ebenso fließend wie die zur gewöhnlichen Sprache. Je nachdem, wie es zu ihrem Gegenstand, ihrem Urheber und der Dialogszene passt. Denn letztlich zielt der Aushandlungsprozess dieser Dialogszene in einer Literaturausstellung doch auf ein einziges Anliegen: Unter ästhetischen Bedingungen und mit den Möglichkeiten der Literalität und Literazität Eindrücke, Gedanken, Gefühle, Ideen, Werte, Verhaltens-, Denk-, Lebensund Arbeitsweisen, soziale und politische System zu verhandeln und überhaupt erst verhandelbar zu machen, die sonst nicht verhandelt werden können. Dazu gehört auch: Widersprüche stehen lassen, der Sprache freien Lauf lassen, abbre-

4 Zur empirischen Untersuchung ästhetischer Negativität vgl. Menninghaus et al. (2017). 
chen. Die Grundvoraussetzung dafür ist, dass Literaturausstellungen nicht als Instrument des Kapitalismus verstanden und unter das Diktat der Masse (der Quote) gestellt werden. Eine Literaturausstellung ist gerade kein Messestand von Mercedes.

\section{Literaturverzeichnis}

Bataille, Georges. Kritisches Wörterbuch. Hg. Maria Kiesow und Henning Schmidgen. Berlin: Merve, 2005.

Brecht, Bertolt. „Fünf Schwierigkeiten beim Schreiben der Wahrheit“. Gesammelte Werke. Bd. 18: Schriften zur Literatur und Kunst 1. Frankfurt a. M.: Suhrkamp, 1990. 222-239.

Demand, Christian. „Kunst und Krempel“. Merkur 72.7 (2018): 18-32. https://volltext. merkur-zeitschrift.de/article/pdf/5b279843536f88a0388b456f/mr_2018_07_00180032_0018_01(23.10.2018).

Graf, Daniel. „Wir Rezensenten. Zum Verhältnis von Buchkritik und Debatte im Zeitalter der Polarisierung“. Republik, 20.10.2018. https://www.republik.ch/2018/10/20/ wir-rezensenten (23.10.2018).

Grant, Nora. How to make a Pop Up Museum. An Organizers' Kit. Hg. Nina Simon. Santa Cruz: Santa Cruz Museum of Art \& History, 2013. http://popupmuseum.org/wp-content/ uploads/2013/09/Pop-Up-Museum-Edited.pdf (23.10.2018).

Lotman, Jurij M. Die Innenwelt des Denkens. Eine semiotische Theorie der Kultur. Hg. Susi K. Frank, Cornelia Ruhe und Alexander Schmitz. Aus dem Russ. übers. v. Gabriele Leupold und Olga Radetzkaja. Berlin: Suhrkamp, 2010.

Menninghaus, Winfried, Valentin Wagner, Julian Hanich, Eugen Wassiliwizky, Thomas Jacobsen und Stefan Koelsch. „The Distancing-Embracing model of the enjoyment of negative emotions in art reception“. Behavioral and Brain Sciences 40 (2017) doi: 10.1017/ S0140525X17000309 (23.10.2018).

Ransmayr, Christoph. „Das Wasserherz“. text+kritik. Christoph Ransmayr 220 (2018): 77-79.

Schalansky, Judith. Verzeichnis einiger Verluste. Berlin: Suhrkamp, 2018. 
